\title{
Electro-Anatomical Approach to Membranous Septal Aneurysm: Potential as a Focus of Critical Ventricular Arrhythmias
}

\author{
Hajime Imura ${ }^{1}$, Hiroshige Murata ${ }^{2}$, Masami Ochi ${ }^{1}$ \\ ${ }^{1}$ Department of Cardiovascular Surgery, Nippon Medical School Hospital, Tokyo, Japan \\ ${ }^{2}$ Department of Cardiology, Nippon Medical School Hospital, Tokyo, Japan \\ Email: himura@nms.ac.jp
}

Received November 8, 2013; revised November 28, 2013; accepted December 5, 2013

Copyright (C 2013 Hajime Imura et al. This is an open access article distributed under the Creative Commons Attribution License, which permits unrestricted use, distribution, and reproduction in any medium, provided the original work is properly cited. In accordance of the Creative Commons Attribution License all Copyrights (C) 2013 are reserved for SCIRP and the owner of the intellectual property Hajime Imura et al. All Copyright (C) 2013 are guarded by law and by SCIRP as a guardian.

\begin{abstract}
Membranous septal aneurysm (MSA) is a rare anomaly and known to cause ventricular tachycardia and atrioventricular block. However, underlying mechanisms have not been addressed in its long history. We report first 3-D electro-anatomical mapping of MSA during and three years following the surgery. An elderly patient underwent a surgery for MSA. In the mapping, we located the His bundle near MSA and observed delayed potentials around MSA. Our report showed that electrophysiological character of myocardium was changed around MSA and this change might be a reason for ventricular tachycardia and atrioventricular block. An ordinary surgery for MSA might not resolve this problem since we still observed delayed potentials three years after the surgery.
\end{abstract}

Keywords: Membranous Septal Aneurysm; Electro-Anatomical Mapping; Ventricular Tachycardia; Atrioventricular Block

\section{Introduction}

The interventricular septum consists of muscular and membranous components. Aneurysm of the membranous portion, which was first described in 1826, is a congenital structure protruding into the right ventricle and can be associated with various pathologies such as rupture, obstruction in the right ventricle and arrhythmias [1]. Ventricular tachycardia (VT) and complete atrioventricular block (AVB) were presented as complications of membranous septal aneurysm (MSA) in old reports [2] and still described as crucial problems in current literatures [3,4], however, only few studies performed electrophysiological investigation for MSA in its long history. To address the underlying mechanisms of such arrhythmias, both anatomical and electrophysiological approaches are indispensable, however, they have not been implemented due to the rarity and difficulty to approach.

A 3-D electroanatomical mapping system (CARTO) constructs a 3-D structure of the heart and draws a col-

\footnotetext{
"Conflict of interest: none declared.
}

or-coded map of impulse propagation and voltage on the figure using electromagnetic technology. The system has been well-established in describing the arrhythmogenic substrate during open-heart surgery as well as catheter ablation for VT. Here we report the first CARTO mapping during and three years following surgery for a case of this rare pathology.

\section{Case Presentation and Scientific Findings}

An elderly patient was referred to our hospital for surgical treatment of MSA. Because MSA's size significantly increased and she became symptomatic (chest discomfort and palpitation), she elected to undergo surgical treatment. She had no other medical history. In preoperative examinations, her resting electrocardiogram showed normal sinus rhythm with no abnormalities. No significant ventricular or other arrhythmias were observed during 24 hours of electrocardiogram monitoring. Also, blood examination yielded no abnormal data. An echocardiogram disclosed a large MSA (3.5 cm in height) while ventricular septal defect was absent. Her coronary 
angiogram showed no stenotic lesions.

Because ventricular septal defect was absent, we performed CARTO mapping (CARTO-XP, Biosense Webster, Diamond Bar, CA, USA) prior to arresting the heart under cardiopulmonary bypass in surgery (Figure 1). In the mapping (Figure 2) MSA exhibited a small amount of electrical activity (fractionated potential), which was obviously from the far field because there were no muscular cells in a histopathological examination (Figure 3). More significantly, we observed delayed potential around MSA (Figure 2). After the mapping, the aneurysm was resected and the defect was closed with a patch. The patient showed uneventful postoperative course and her
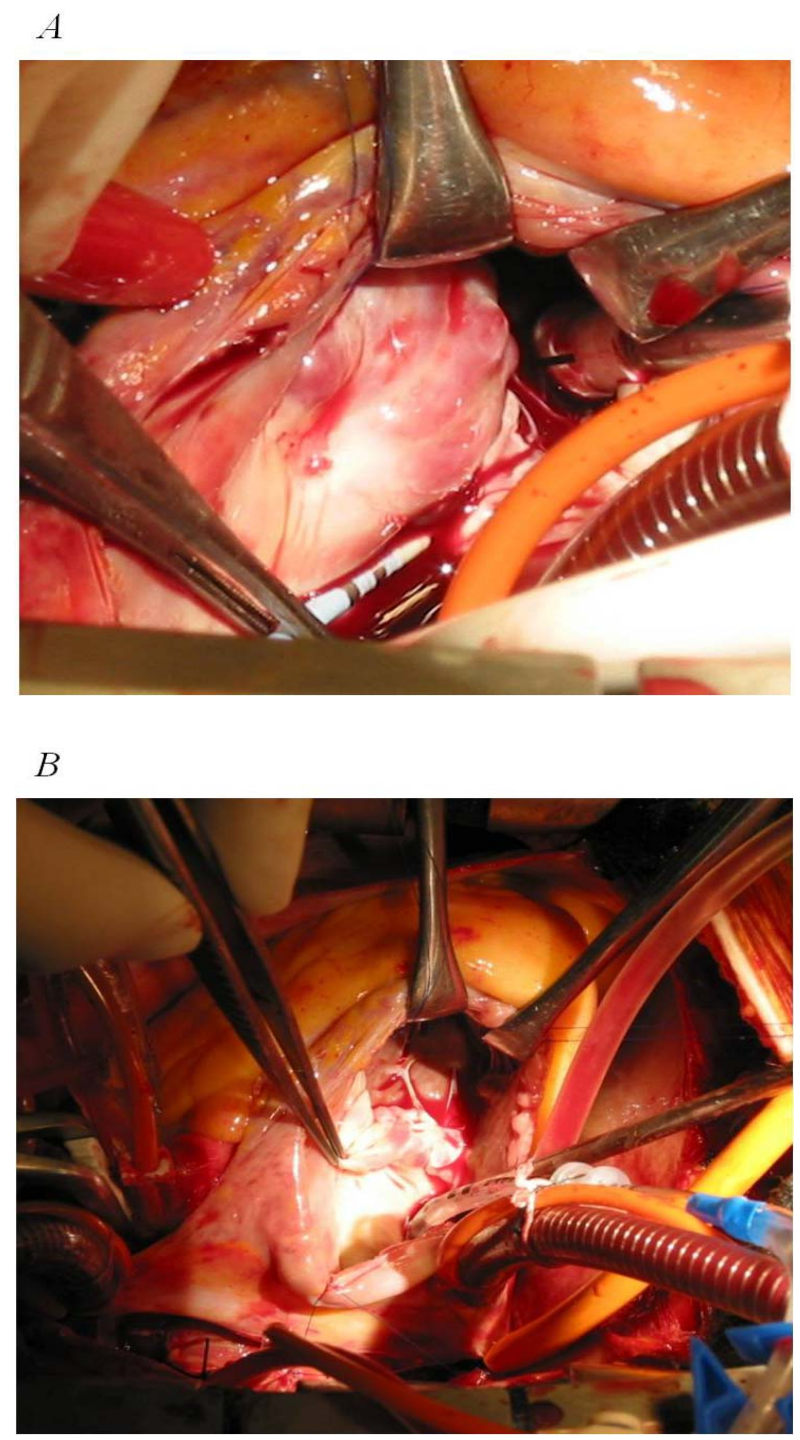

Figure 1. (A) Membranous septal aneurysm (MSA) with the heart beating. The MSA was extremely large between the anterior and septal leaflets of the tricuspid valve and bulges into the right ventricle. A catheter was placed near the His bundle. (B) MSA under cardiac arrest. A chordae-like structure was seen. symptoms were improved, however, the delayed potential was still observed three years after the surgery (Figure 4).

\section{Discussion}

This report is the first to show a 3-D voltage mapping and approach the mechanism of arrhythmias in MSA. We observed delayed potential around MSA, indicating that MSA could be a focus of VT [5]. The evaluation became possible by placing a catheter all around MSA without any restrictions under cardiopulmonary bypass, and we for the first time recorded delayed potentials around MSA. The cause of delayed potentials was unclear. It is well known that left ventricular apical aneurysm is sometimes accompanied by delayed potentials in the myocardium around it and can be a focus of VT [6]. Because of the structural resemblance between MSA and left ventricular apical aneurysm, similar mechanisms can
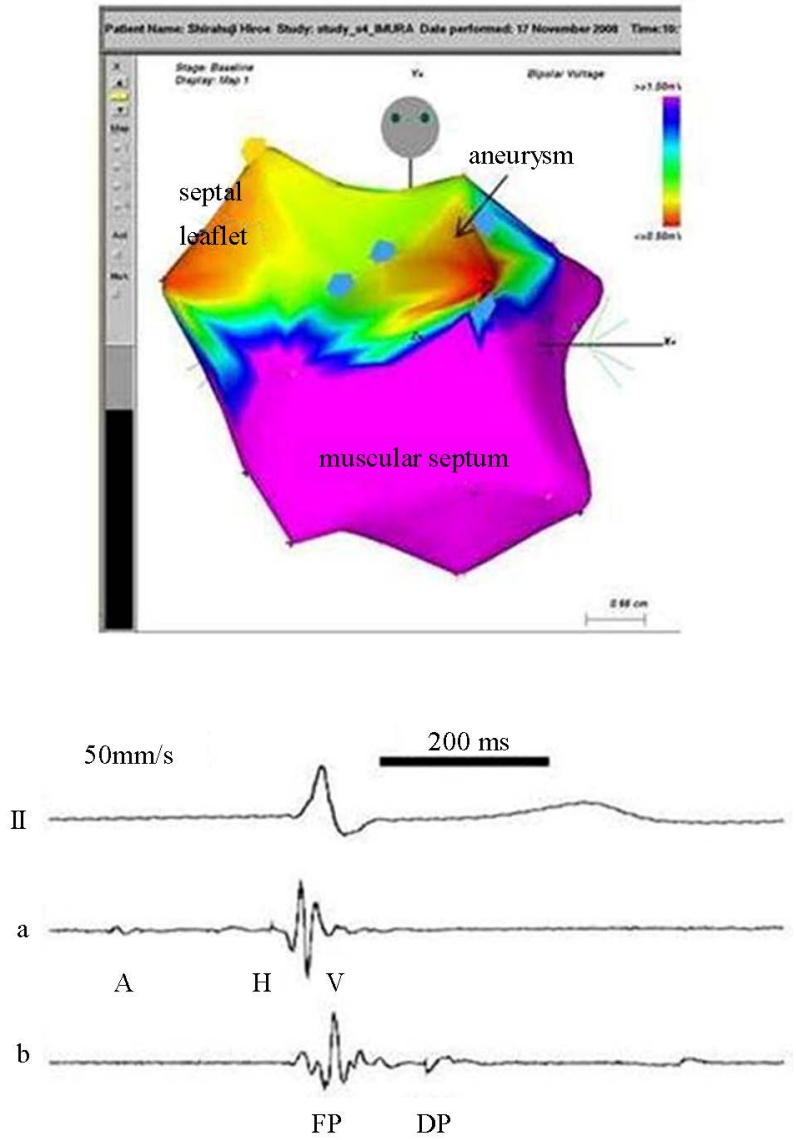

Figure 2. (Upper Panel): A schematic representation of the electro-anatomical mapping during surgery (anterior view). The color changes according to electrical potential (red-blue: 0.5 - $1.5 \mathrm{mV}$ ). Blue tags identify points where the delayed potential was observed and yellow tags shows the His bundle. (Lower Panel): Electrical activities observed in II lead, at the yellow tag (a), and blue tags (b). FP: fractionated potential. DP: delayed potential. 

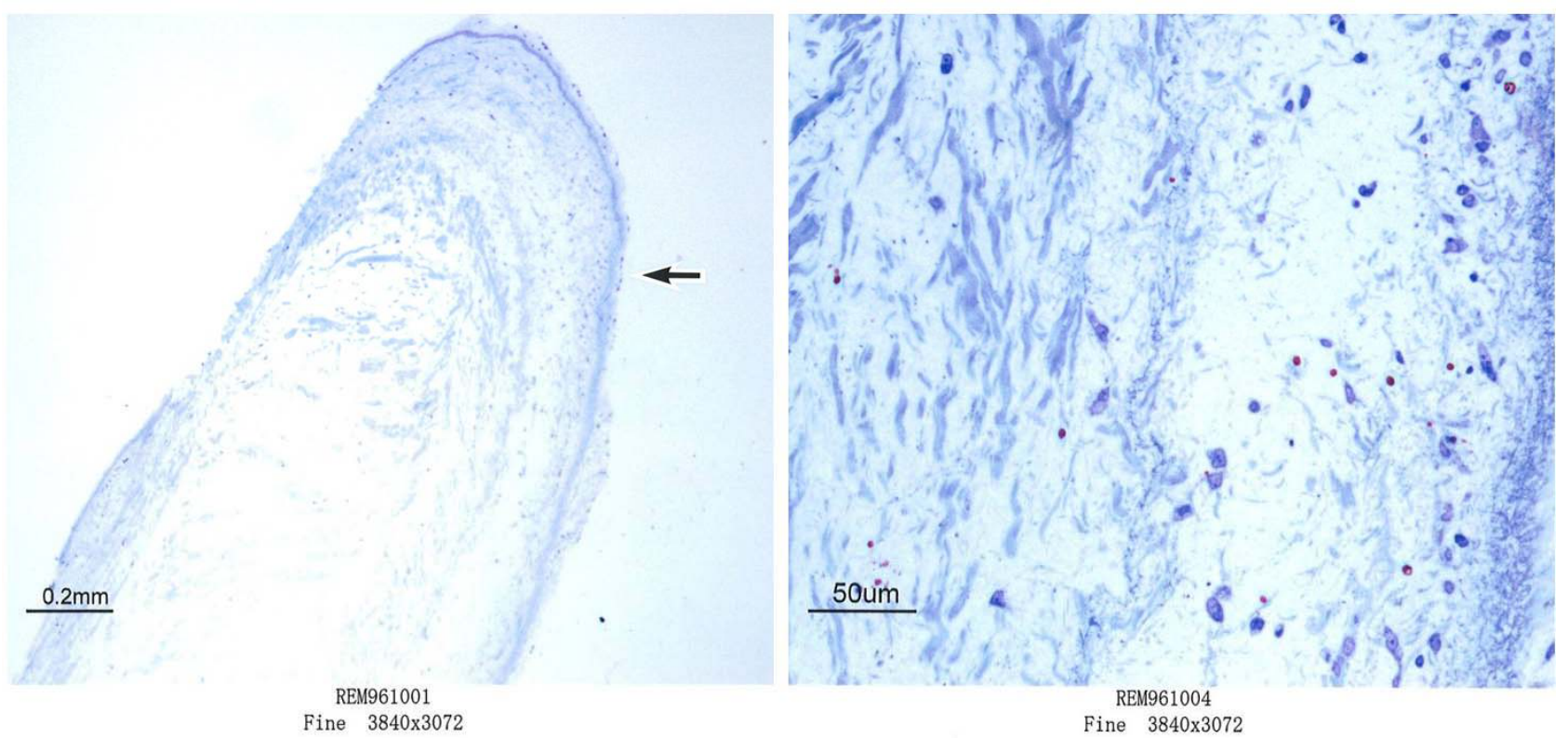

Figure 3. (A) Light microscopy examination stained with toluidine blue. An elastic lamina-like structure is visible along the right margin. The tissue mainly consists of myxoid matrix. Areas where elastic fibers are abundant are partially observable. (B) An electron microscopy examination of tissue indicated by an arrow. Smooth muscle cells, collagen fibers and elastic microfilaments are seen underneath the endocardial cells. A muscular component was not seen in both (A) and (B).

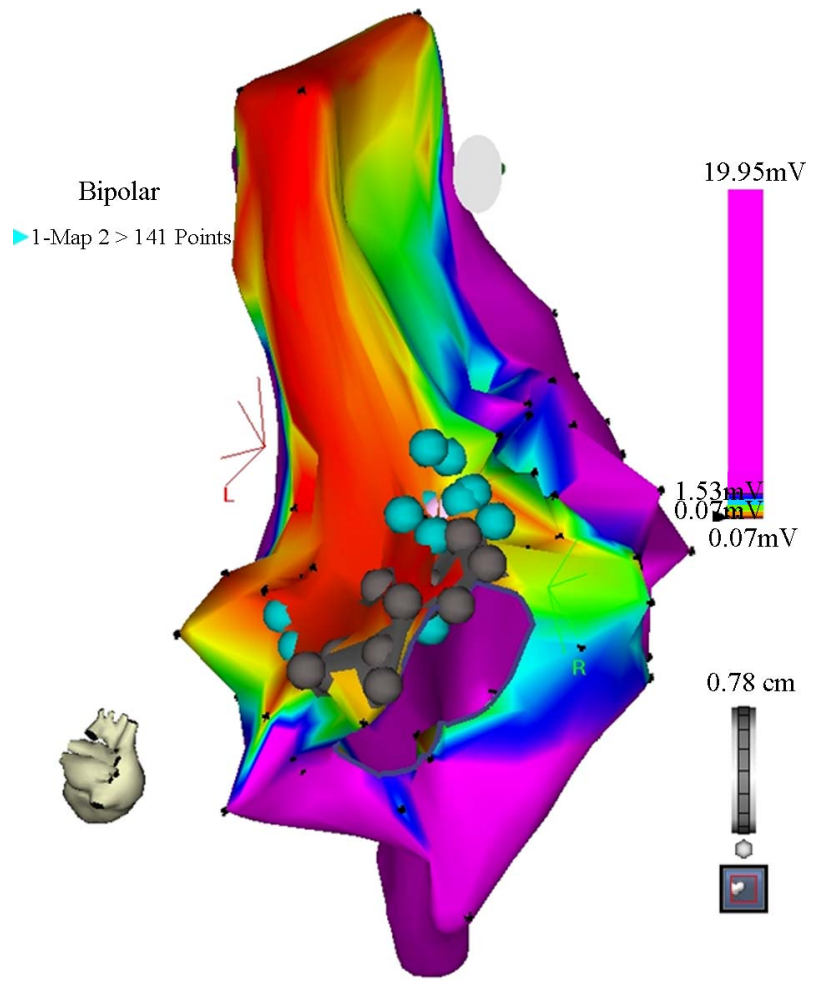

Figure 4. A schematic representation of the electro-anatomical mapping three years after the surgery. The schema presents a right lateral view of the mapping. Blue tags identify points where the delayed potential was observed and black tags shows the patch. Delayed potentials were still observed around the patch. be at work for MSA. Thus, we speculate that stretching force due to left ventricular pressure around MSA may be a primary reason of these delayed potentials and can cause VT for MSA. The force may also be the cause of AVB because the His bundle was near MSA.

As previous studies recommended [7], the aneurysm was resected and the defect was closed with a patch for this case. We placed all the sutures on the myocardium (except near the His bundle and tricuspid annulus) to reduce the stretching force, nevertheless, the delayed potential was still observed three years after the surgery. Persistent stretching force over the long term may cause irreversible changes in the electrophysiological character of the myocardium around MSA

\section{Conclusion}

We experienced a surgery for large MSA, which was known as potential causes of critical arrhythmias such as VT and AVB. Although the patient had not shown such arrhythmias, for the first time, we observed changes in the electrophysiological character of the myocardium around MSA. These changes can be an evidence of predisposition to VT and AVB for MSA. Thus, we approached to the mechanisms of critical ventricular arrhythmias in MSA, which have not been addressed since this pathology was first presented. Persistent stretching force over the long-term may cause irreversible changes in the electrophysiological character of the myocardium around 
MSA because the delayed potential was still observed three years after the surgery.

\section{REFERENCES}

[1] R. I. Hamby, F. Raia and O. Apiado, "Aneurysm of the pars Membranacea. Report of Three Adults Cases and a Review of the Literature," American Heart Journal, Vol. 79, No. 5, 1970, pp. 688-699. http://dx.doi.org/10.1016/0002-8703(70)90289-9

[2] R. J. Clark and P. D. White, "Congenital Aneurismal Defect of the Membranous Portion of the Ventricular Septum Associated with Heart Block, Ventricular Flutter, Adams-Stroke Syndrome and Death,” Circulation, Vol. 5, No. 5, 1952, pp. 725-729.

[3] A. Graffigna, G. Minzioni, L. Ressia, et al., "Surgical Ablation of Ventricular Tachycardia Secondary to Congenital Ventricular Septal Aneurysm,” The Annals of Thoracic Surgery, Vol. 57, No. 4, 1994, pp. 921-924. http://dx.doi.org/10.1016/0003-4975(94)90203-8
[4] S. W. Jang, T. H. Rho and J. H. Kim, "Membranous Interventricular Septal Aneurysm Resulted in Complete Atrioventricular Block,” Heart, Vol. 96, No. 3, 2010, p. 244. http://dx.doi.org/10.1136/hrt.2009.175588

[5] W. G. Stevenson and K. Soejima, "Catheter Ablation for Ventricular Tachycardia,” Circulation, Vol. 115, No. 21, 2007, pp. 2750-2760. http://dx.doi.org/10.1161/CIRCULATIONAHA.106.6557 $\underline{20}$

[6] H. Furushima, M. Chinushi, K. Iijima, et al., "Ventricular Tachyarrythmia Associated with Hepertrophic Cardiomyopathy: Incidence, Prognosis, and Relation to Type of Hypertrophy,” Journal of Cardiovascular Electrophysiology, Vol. 21, No. 9, 2010, pp. 91-99. http://dx.doi.org/10.1111/j.1540-8167.2010.01769.x

[7] B. A. Vidne, L. Chiariello, H. Wagner, et al., “Aneurysm of the Membranous Ventricular Septum. Surgical Consideration and Experience in 29 Cases," The Journal of Thoracic and Cardiovascular Surgery, Vol. 71, No. 3, 1976, pp. 402-409. 